UDC 930.85(450 Trst)

821.131.1-992

DOI 10.18485/ITALBG.2016.2.1

HTTPS://DOI.ORG/10.18485/ITALBG.2016.2.1

\author{
Marija Mitrović* \\ Università degli Studi di Trieste
}

\title{
CITTÀ DI CARTA
}

\begin{abstract}
Nel saggio si rintraccia il lungo percorso che ha portato a Trieste il titolo città di carta; successivamente si confronta l'immagine letteraria di Trieste con quella di Belgrado. Nel costruire e costituire il profilo letterario della città di Trieste e nel porre la stessa città sul gradino più alto tra $\mathrm{i}$ centri culturali del Novecento, hanno contribuito gli scrittori, ma senza un supporto "logistico" forte da parte degli storici e dei critici letterari non lo si poteva fare (Ara, Magris, Fölkel, Guagnini). Negli ultimi decenni l'identità plurima della città è diventata visibile. Solo liberandosi dai miti e dalle leggende la cultura triestina ha raggiunto livelli invidiabili e oggi si può misurare con delle città che alle spalle hanno storie molto più ricche e più lunghe. I libri di storia e storia culturale della città di Belgrado, invece, partono sempre da tempi molto lontani; sono documentati da scarni reperti archeologici e da testimonianze legate ai miti e alle leggende (Velmar-Janković, Pavić, Pejčić). In assenza di una storia culturale moderna, mettiamo al centro del nostro interesse i romanzi che posano lo sguardo critico verso la storia culturale di questa città (Uskoković, Matavulj, Andrić, Crnjanski, Velmar-Janković, Selenić, Pantić, Pištalo).

Parole chiave: Trieste, Belgrado, storia culturale moderna, letteratura, demitizzazione, identità plurime.
\end{abstract}

L'immagine della città creatasi con delle parole, presentata nei testi saggistici e letterari in senso più stretto, ha nel processo di ricezione da parte del pubblico il valore e l'importanza pari all'immagine che sulla stessa città viene fuori dai documenti storici, dall'aspetto architettonico oppure dall'arte visiva. Dire per una città che è una città di carta è un complimento alto ed essenziale: è una garanzia che l'immagine letteraria in questione è complessa, veritiera, affidabile. Dagli ultimi decenni del Novecento, in varie occasioni e nei titoli di alcuni contributi storico-antropologici, la città di Trieste è stata nominata proprio così: la città di carta. Ci sembra utile rintracciare, a grandi linee, il lungo percorso che ha portato a questa città un titolo così lusinghiero. Inoltre, questo saggio si pone uno scopo ancora più

*marmitrovic41@gmail.com 
complesso, quello di confrontare l'immagine letteraria di Trieste con quella di Belgrado. Con quale motivazione? Che cosa ci permette un confronto così rischioso che comunque può essere solo approssimativo? Le motivazioni non partono dalle somiglianze storiche, antropologiche, sociali tra queste due città (seppure queste esistano e alcune saranno menzionate più avanti), ma da un voto antico, che sta agli albori della letteratura e cultura moderna serba. Più di due secoli fa il primo scrittore moderno serbo Dositej Obradović aveva posto Trieste come esempio positivo, come un parametro e un modello da seguire, pensando alla città di Belgrado, appena liberata. Sul ruolo della cultura italiana ${ }^{1}$ e sul ruolo di Trieste nel programma volto al futuro sviluppo di Belgrado e della Serbia che avrebbe formulato Dositej come primo ministro dell'educazione serbo, ho scritto altrove ${ }^{2}$. Qui vorrei porre l'accento sul fatto che nell'ultimo capitolo del libro Mezimac (pubblicato postumo nel 1818), Dositej nota già l'esistenza di "uno stato ideale" seppur minimo. Egli scrive:

Non ci si arrende alla superbia, né alla presunzione, né alla discordia, cattiva madre
di tutti i mali, ma con saggia calma e con virile e generoso amore per la giustizia
e il patriottismo si celebra la potenza e la misericordia dell'Onnipotente; si vuole
e si tende, ponendo in ciò tutta la propria ambizione, che tra i popoli gloriosi - an-
ch'esso diventi glorioso, coraggioso, impavido, illuminato, amante della giustizia,
filantropo e benefattore ${ }^{3}$ (traduzione mia).

"Glorioso tra i popoli gloriosi" a quel tempo era soltanto il popolo della "nazione illirica” di Trieste! Solo esso possedeva un' eccellente organizzazione, che allora era desiderosa di una sempre più completa istruzione (non dimentichiamo che sotto il concetto di "scuola" nella seconda edizione del 1852 del suo Rječnik, Vuk Stefanović Karadžić scrisse che la migliore scuola dell'Impero Austriaco era proprio quella serba di Trieste!) e che la comunità illirica già allora aveva 1'abitudine di favorire con azioni benefiche tutto quello che necessitava di un aiuto. Sarebbe illusorio immaginare tali peculiarità in una comunità che all'epoca combatteva per la liberazione come quella della Serbia, la quale, tra l'altro, Dositej non conosceva ancora di prima mano. L'immagine positiva della comunità che pone come esempio

\footnotetext{
${ }^{1}$ Bonazza (1990)

${ }^{2}$ Mitrović (2008, 2009a, 2009b, 2011, 2013)

3 “Ne predaje se gordosti, ni visokoumiju, ni neslogi, zloj sviju zala materi, nego s trezvenim smirenijem i s mužestvenim i velikodušnim pravdo- i otečestvoljubijem proslavlja silu i milost svemogućega; želi i nastoji, i u tome sve svoje slavoljubije postavlja, da među slavni narodi slavan, hrabar, neustrašim, prosvešten, pravdoljubiv, čelovekoljubiv i dobrodetelen postane" (Obradović 2008: 185).
} 
per la futura nazione serba era proprio quella in cui allora, all'inizio del XIX secolo, aveva dimorato, cioè la comunità illirica di Trieste.

Mettiamo qui a confronto le immagini letterarie di due città, quella di Trieste e quella di Belgrado, per vedere se e in che modo possiamo ancora oggi, oltre due secoli dopo Dositej, trarne dei vantaggi seguendo le tracce di quella che si è guadagnata il titolo di città letteraria.

Tutti quelli che conoscono la storia di Trieste, soprattutto la parte che riguarda il periodo successivo alla prima guerra mondiale, nel quale da porto principale di un impero, la città diventò l'estrema periferia del regno italiano e presto scivolò verso il fascismo, sapranno senz'altro che la positiva immagine culturale della città si costruiva con fatica e con molti ristagni. Finché alla storiografia si prediligeva il mito nazionalista sulla citta "italianissima", si sapeva e si poteva leggere poco sulla letteratura triestina. Nel costruire e costituire il profilo letterario della città di Trieste e nel porre la stessa città sul gradino più alto tra $\mathrm{i}$ centri culturali del Novecento, hanno contribuito sicuramente gli scrittori, ma senza un supporto "logistico" forte da parte degli storici e dei critici letterari non lo si poteva fare. È importante tener presente la complessità della lunga e tormentosa salita verso questa posizione così alta, altrimenti si potrebbe rimanere sorpresi ed increduli davanti al fatto che "nel volumetto divulgativo-erudito di Baccio Ziliotto, Storia letteraria di Trieste e dell'Istria (Trieste, 1924), tentativo di tracciare un quadro complessivo della cultura giuliana esteso dalle origini al Novecento" (Guagnini 2015: 304) non compariva il nome di Italo Svevo, sebbene lui avesse già pubblicato tutti $\mathrm{e}$ tre i suoi romanzi più noti: Una vita (1892), Senilità (1898), La coscienza di Zeno (1923). Non fu menzionato neanche Umberto Saba, sebbene il Canzoniere fosse stato pubblicato nel 1922. Sarebbero dovuti trascorrere parecchi anni prima di poter giungere alla comprensione della specificità e del valore della poetica letteraria triestina, impregnata già all'inizio del Novecento con problemi psicologici e con analisi dettagliate della vita interiore dei protagonisti.

Solo in seguito all'intervento svolto dagli studi storici, i quali hanno fatto luce sul rapporto complesso tra gli italiani e la più numerosa minoranza nazionale di Trieste e dopo che $\mathrm{i}$ critici letterari hanno presentato al pubblico italiano i contributi letterari scritti in diverse lingue e linguaggi, la città ha assunto le coordinate necessarie per potersi fregiare del titolo di città di carta. La nota distintiva degli scritti composti all'interno di questa zona culturale corrisponde alla raffinatezza stilistica che qui sarà illustrata con un frammento della prosa di Claudio Magris, uno degli autori che - come critico letterario e culturale - ha contribuito maggiormente alle analisi precise del profilo culturale della città. Il frammento preso dal libro 
Microcosmi (1997) si ritiene paradigmatico di questo nuovo modo di presentare la storia della città:

La schiuma scende, si allunga in chiazze bavose, sparisce in uno scolo ai margini del Giardino e intanto da San Giovanni un Vodopivec scende o sale in città e diventa Bevilacqua. Il Patok fluisce dalla Slavia al Mare Nostrum, l'Italia si fa crogiolo anche di chi viene da lontano e assai presto si sente italiano come chi porta un cognome veneto o friulano; le giovinezze che nella grande guerra vanno a farsi recidere sul Carso affinché Trieste si unisca all'Italia si chiamano Slataper, Xidias, Brunner, Ananian, Suvich. Ma il crogiolo si rapprende, gli elementi si scambiano e contrappongono; la città-frontiera è intessuta e tagliata da frontiere che la dividono da se stessa, cicatrici che non rimarginano, confini invisibili e fatali fra una pietra e l'altra del selciato, violenze che chiamano violenze. Quel rigagnolo è rossastro, la storia ha le mestruazioni; una volta tocca a me una volta a te, comunque in quell'acqua limacciosa un sangue non si distingue da un altro (Magris 1997: 239).

Trieste è un anacronismo e un nebeneinander, una spiaggia affastellata di detriti della Storia, in cui tutto è il contrario di tutto coesistono a contatto di gomito, irredentismo e fedeltà absburgica, patriottismo italiano e cognomi tedeschi e slavi, Apollo e Mercurio. In quel cul di sacco dell'Adriatico la Storia è un gomitolo i cui fili si aggrovigliano (Magris 1997: 245).

Se lo spazio descritto nel romanzo Danubio (1986) fu enorme, dalla sorgente alla foce del fiume Danubio, nel libro degli appunti di viaggio molto specifici e dal punto di vista dei generi letterari difficilmente qualificabili - in Microcosmi, come dice già lo stesso titolo, lo spazio è estremamente limitato, si concentra su un chilometro quadrato, compreso tra la casa del narratore e il suo caffè preferito, tra il parco pubblico e la vicina chiesa. "Il viaggio" si confronta poi con le immagini e le scene riferite a spazi non meglio precisati, ma comunque situati "dall'altra parte", nel paese dove gli slavi sono la maggioranza degli abitanti. Tenendosi stretto a questo minispazio e intrecciando il passato con il presente, Magris è riuscito ad accennare alla struttura complessa della propria città e a sottolinearne la sua identità plurima. La larghezza di vedute dalle quali è partito (la conoscenza dettagliata della cultura, letteratura, storia e filosofia tedesca e quella europea in genere), le attualità socio-politiche che segue e commenta nei testi pubblicistici dei principali giornali italiani di oggi, la dote narrativa che ha raffinato, non solo scrivendo, ma anche tenendo lezioni, tutto questo insieme ha abilitato Magris a scrivere una mappa vivace e tangibile della complessità della cultura triestina: non sono presenti solo i nomi e i temi più importanti che fanno parte della cultura triestina, ma si avverte forte e nitido anche il tono ironico verso il mito nazionale come anche la vicinanza dell'autore alle anime dei fondatori della modernità. Le immagini di filigrana che riguardano la mentalità dei triestini hanno permesso all'autore di illustrare 
molte specificità dell'identità della sua città, come anche di intrecciare tanti cambiamenti susseguitisi lungo gli ultimi due secoli. Davanti agli occhi del lettore si trovano sia i dettagli minimi sia le larghezze cosmiche, lo spazio limitato in un tempo prolungato: il tempo reale non supera il passaggio di un bambino preoccupato per il destino di un pesciolino che lui porta in un vassoio dalla sua casa verso il laghetto del parco, cercando di salvare la sua vita restituendolo all'acqua torbida del laghetto, mentre il tempo simbolicamente presente e rievocato in vari modi si riferisce a due secoli della storia culturale e politica della città. Senza la retorica, con le emozioni e tanto calore, ma anche con l'ironia, Magris riesce a creare un capolavoro letterario sulla storia culturale della sua città.

La descrizione di questo quadro così complesso è stata possibile grazie anche agli studi dettagliati del passato triestino. E tra i libri che oggi ogni triestino di qualsiasi appartenenza etnica o culturale legge volentieri, compare proprio il libro scritto a quattro mani dallo storico Angelo Ara e lo scrittore Claudio Magris, intitolato Trieste, un'identità di frontiera, pubblicato per la prima volta nel 1982, il quale ad oggi è giunto alla sua quinta edizione.

Il capitolo finale del libro di appena duecentootto pagine e ricco di fatti e scritto in uno stile saggistico attraente, è intitolato Città di carta. La frase finale del capitolo e dell'intero libro recita così: "Una condizione che si può vivere, ma non predicare: 'quando poi qualcuno viene - scriveva già Slataper a Sibilla Aleramo nel 1912 - noi non sappiamo fare altro che condurlo per queste grigie vie e meravigliarci che egli non capisca"' (Ara \& Magris 1982: 208). La frase scritta da Scipio Slataper (1888-1915) più di cento anni fa e nota anche oggi, forse non è più collegata tanto con la città di Trieste, ma con quella di Belgrado e - più in generale - con la Serbia: tante volte siamo propensi a lamentarci del fatto che "gli altri" non ci comprendono, che siamo fraintesi... Gli autori che avevano scritto sull'identità specifica della città di Trieste facevano tutti gli sforzi per chiarire e depurare il canale comunicativo dove da tanto tempo si sentivano i rumori. Forse anche la letteratura che cerca di presentare la storia della città di Belgrado - dove tuttora si scorge il rumore nel canale comunicativo - potrebbe ravvisare le chiavi per la diminuzione di questa incomprensione tra "noi" e "gli altri", tra l'immagine storico-culturale che noi stessi costruiamo e quella che su di noi creano gli altri.

Già all'inizio del Novecento Hermann Bahr aveva indicato Trieste come "nessun posto - meraviglioso, più bello di Napoli, però non è una città. Si ha l'impressione di non essere in alcun posto" (Ara \& Magris 1982: 187). Verso la metà del secolo scorso Enzo Bettiza (Split, 1927) presentò questa città come fantasma (Il fantasma di Trieste, 1958). Toni diversi invece nei testi di Ernestina Pellegrini, italianista e comparatista dell'Università di 
Firenze che nell'anno 1987 pubblicò il libro La Trieste di carta. Qualche anno dopo (2002) Pellegrini ha rivisto e sintetizzato queste sue ricerche, pubblicando un capitolo sul libro Storia d'Italia. Le Regioni, dall'unità ad oggi, Friuli Venezia-Giulia con il titolo La città di carta. Lo stesso dunque che fu usato per nominare l'ultimo capitolo del libro di Ara e Magris. Ed è da allora che questo marchio fa rima con la città di Trieste. Nel testo della Pellegrini si legge:

$\mathrm{Ci}$ sono alcuni elementi di persistenza nella raffigurazione, come se l'immagine nuova si producesse sempre a ridosso dell'immagine precedente in un sovrapporsi di pennellate e cancellature, creando un'aria di famiglia, una leggenda scritta che allontana il dato documentario è l'originale in un passato irrecuperabile o irrilevante (Pellegrini 2002: 1207).

Sentiamo ora come all'inizio del Novecento fu vista questa città che oggi sembra costruita dalle parole e dalle storie che sorgono una dall'altra: "Trieste non ha tradizioni di cultura" ha scritto Scipio Slataper nel 1909 sulla rivista fiorentina La Voce, dove all'epoca collaboravano gli studenti triestini che preferivano l'Università di Firenze alle università della monarchia asburgica. Il libro di Ara e Magris inizia proprio con Slataper e con la sua dichiarazione estremamente critica:

Nel Mio Carso Scipio Slataper confessa ed esorcizza nei primi tre capoversi che iniziano tutti con le parole "vorrei dirvi", una tentazione di mentire. Egli vorrebbe dire ai suoi lettori, e cioè agli italiani, di essere nato in una casupola sul Carso oppure in una foresta di roveri in Croazia o nella pianura morava; egli vorrebbe dar loro ad intendere di non essere un italiano e di aver solo "imparato" quella lingua in cui scrive, e che non gli piace bensì gli ridesta "il desiderio di tornare in patria perché qui sto molto male" (Ara \& Magris 1982: 3).

Usare come incipit del libro una frase che esprime solo una brama (o forse addirittura una menzogna!) sull'identità etnico-spaziale plurima dell'autore il quale, allo stesso tempo, nega l'esistenza della cultura triestina, dice molto al lettore; gli dice soprattutto che il libro non ha l'intenzione di nascondere niente, di non voler abbellire il passato, che si soffermerà sui punti negativi e dolenti della storia triestina. Perché è l'unico modo di superare i problemi; è questa la tecnica con la quale il libro incanta il lettore e solo così diventa un libro di grande importanza nello studio sull'identità triestina. Gli autori colgono ogni occasione per decomporre i miti che girano attorno alla storia della città. Così si viene a sapere che per secoli nella città hanno vissuto diversi popoli e le loro rispettive culture, le quali però non s'intrecciavano e dunque non potevano neanche arricchirsi reciprocamente. Erano presenti nebeneinander (gli autori preferiscono usare questo 
termine tedesco), e non invece mescolate tra di loro. I risultati perciò erano magri, la scrittura maggiormente epigone. Il che ha provocato Slataper a formulare un giudizio assolutamente negativo sulla (non) esistenza della cultura triestina.

Il libro di Ara e Magris si apre con fatti del passato di cui la città non può vantarsi. Bisognava presentare tutta la storia prima di giungere al periodo nel quale è divenuto possibile realizzare quello che per Slataper rappresentava pura brama. Solo negli ultimi decenni l'identità plurima della città è diventata visibile e realizzabile così che solo oggi la frase che nega la presenza della cultura alla città di Trieste non si legge più come una pesante accusa, ma come un segno, uno spartiacque tra la vecchia cultura epigone e quella nuova, originale e importante non solo per la letteratura italiana, ma addirittura per quella europea. L'aspra negazione ha stimolato gli scrittori e gli altri artisti ad impegnarsi nella creazione di una cultura autentica e moderna.

Solo un anno dopo la pubblicazione del libro Trieste, un'identità di frontiera, esce Trieste provincia imperiale (1983) di Ferruccio Fölkel e Carolus Cergoly. "Nato a Trieste nel 1921 e scomparso a Grado (Gorizia) nel 2002, Ferruccio Fölkel ha vissuto gran parte della sua vita a Milano, dove si era stabilito nel 1957 per svolgere la professione di redattore editoriale, collaborando prima con Alberto Mondadori, poi con la Mondadori stessa, nella quale fin dalla fondazione nel 1965 lavorò alla celebre collana economica degli «Oscar»" - si legge in un testo di Anna Millo (2007: 80, 81), che sottolinea:

Fölkel è una voce sempre razionalmente critica verso le semplificazioni e le affabulazioni del mito letterario triestino. [...] Nella sua ricerca intellettuale non si lascia trasportare da trasfigurazioni fantastiche, ma resta aderente, per la sua cultura d'impronta storicistica, alla realtà storica e sociale che non rinuncia mai ad indagare. In questo compito non si trovava certo facilitato dalle condizioni in cui versavano gli studi storici di argomento locale, a lungo irretiti anch'essi nelle spire di un mito, il mito nazionalistico della città «italianissima» che per venti secoli, dalla latinità alla nazione ottocentesca, attende di essere ricongiunta alla sua madrepatria, mentre è acquisizione storiografica relativamente recente una visione della cultura italiana della città come risultato di una sintesi di elementi diversi, di un processo sofferto e non lineare.

A differenza del libro di Ara e Magris scritto "a quattro mani" senza che si sappia esattamente chi ha scritto quale capitolo del libro, qui l'Indice mostra che la gran parte del libro è stata opera di Fölkel (circa duecentotrenta pagine) mentre Cergoly è l'autore di una sessantina di pagine. La nostra attenzione si sofferma sulla parte scritta da Fölkel. Se il libro di Ara e Magris 
ammonisce esplicitamente che i miti e le leggende spesso s'inseriscono nelle narrazioni sulla città, qui è già la stessa sintassi impregnata da questo sapere come i miti e le leggende non aiutano a capire la vera storia. Nelle frasi che Fölkel usa per descrivere il passato di Trieste domina il condizionale, il sintagma "dicono che", l'avverbio "forse", mentre la narrazione intrecciata attorno al passato di Trieste è indicata come "cosiddetta storia". La scrittura stessa di Fölkel (Fölkel \& Cergoly 1983: 16) comunica al lettore che non c'è niente di fermo e compatto, niente di apodittico: "Fu la lasciva Aquileia a costituire il maggior ostacolo al progresso della modesta Trieste? Può darsi. Da Aquilea giunsero - mito o storia? - i primi predicatori cristiani". Dicono che tra questi era anche il protettore di Trieste, San Giusto: "ma San Giusto emerge appena da un documento del 948" (ibid.). E così via. Per questo autore, deciso a sfatare i miti, la storia è solo quello che si basa sui documenti.

Oppositore deciso di tutti i nazionalismi ${ }^{4}$, Fölkel si è dimostrato molto abile nel collegare tra di loro le storie sociali, economiche e culturali della città di Trieste e ad elencare i fatti che rendevano questa città un emporio commerciale e culturale importante; non ha dimenticato neanche gli errori della politica viennese verso Trieste non solo durante la prima guerra mondiale, ma anche molto prima. Ha inoltre dedicato un'attenzione particolare alla vita quotidiana della città composta di gente arrivata da molte parti del Mediterraneo, ma unita dal desiderio di iniziare una nuova vita, di arricchirsi ad ogni costo. C'era tanta ipocrisia e sospetto soprattutto tra gli intellettuali: Ettore Schmitz, cioè Italo Svevo, non era riconosciuto come scrittore anche perché non era concepibile che un membro della famiglia degli industriali benestanti "perdesse" il tempo scrivendo la prosa! L'Autore si è a lungo soffermato sul comportamento della gente d'affari durante gli spettacoli teatrali in scena nel primo grande teatro triestino, inaugurato nel 1801. Come documento ha citato un frammento degli appunti di viaggio del viaggiatore tedesco, Johann Gottfrid Seume, che nel 1802 ha viaggiato sulle orme di Goethe e si è soffermato prima a Trieste per cercare la tomba di Winckelmann, grande archeologo tedesco ucciso proprio in questa città; non ha trovato nessuno che gli potesse dare qualche indicazione né sul delitto, né sulla tomba. Ed ecco come Seume descrive il pubblico triestino: "Qui per

4 "Da quel mondo della musiliana Kakania che non riusciva a stare al passo con $\mathrm{i}$ tempi, da quel mondo che non seppe trasformarsi, emersero le nazionalità, e dunque prese forma e consistenza il nazionalismo, una peculiare 'bestialità' dell'uomo. E l'Adriatico vide sorgere e poi affermarsi due irredentismi di segno opposto, talvolta violenti, incongrui, eppure complementari: l'italiano e lo slavo. Ma tutta l'Europa fu infetta dal tremendo male. Tanto che la decadenza del vecchio continente procedette di pari passo con lo scatenarsi del virus nazionalista" (Fölkel \& Cergoly 1983: 44). 
la prima volta ho udito lo strepitìo assordante dei teatri italiani. Si approfitta dello spettacolo, per tener conversazione, per trattare affari di borsa, e per chi sa quante altre cose. Soltanto le arie predilette vengono ascoltate con compunzione, ma per il resto un devoto di Talia ha ben scarsa la possibilità di godersi la musica" (Fölkel \& Cergoly 1983: 115). Fölkel descrive poi la situazione di trent'anni dopo, che ancora non era cambiata:

La gente parla, discute di affari, le signore esibiscono alcune toilets con riferimenti viennesi, probabilmente. Accade che una brava cantante, stanca di esibirsi nel più assoluto disinteresse verso la cultura, si volta e presenta al pubblico le sue parti posteriori, dopo essersi ben piegata e aver sollevato le vesti. I triestini non se ne accorgono subito, tale è l'accanimento dei loro mercanteschi discorsi; quando, però se ne accorgono, da perfetti ipocriti, provocano uno scandalo e per poco la brava cantante non finisce all'Intendenza di polizia. L'intendente è tuttavia un viennese di grande spirito che in cuor suo soffre per l'atteggiamento degli emporiali triestini. Così non soltanto mette a tacere il presunto scandalo ma riesce a far partire indenne l'amante di Talia... (ibid.).

Sono molti gli argomenti che Fölkel ha toccato, sempre in modo convincente e argomentato: quali erano le conseguenze a livello urbanistico e sociale dello sviluppo urbanistico veloce (da una cittadina di trentacinquemila abitanti, in cento anni si trasforma in una città di duecentotrentamila residenti), ma soprattutto come si comportava la gente a livello culturale. Cita una lettera di Joyce che riguardo alla Trieste della sua epoca si esprime così: "È impossibile esagerare la scortesia della gente. Donne e ragazze sono così villane che Nora ha paura di uscire per strada" (Fölkel \& Cergoly 1983: 129).

D’altra parte, era proprio Fölkel che per primo dedicò pagine encomiabili alla poesia di Srečko Kosovel, poeta sloveno nato a Sežana, sconosciuto per molto tempo ai triestini e alla letteratura italiana nonostante i francesi lo avessero già scoperto in precedenza e avessero già pubblicato le sue opere: "Una scoperta che mi ha dato grandissima gioia. Anche un senso di pena, per averlo letto così tardi. E in italiano. Perché, si sa, il triestino emporiale non conosceva che l'italiano e il triestino. E io mediocremente l'italiano, male triestino" (Fölkel \& Cergoly 1983: 146)

${ }^{5}$ La famiglia Fölkel era di origine ebraica, proveniva dalla Galizia, i nonni hanno vissuto a Trieste e poi a Budapest, mentre il padre si è cristianizzato nell'Italia meridionale. Ferruccio Fölkel invece era un laico che a lungo e molto profondamente si occupò di temi sull'ebraismo; era proprio lui uno dei primi a scrivere gli studi sulla risiera di San Sabba, lager nazista situato a Trieste, usato per il transito, detenzione ed eliminazione di prigionieri politici nonché di numerosissimi ebrei. 
Il libro Trieste provincia imperale si legge e si cita spesso, ma è comunque significativo che non fu mai ristampato e oggi è reperibile solo presso le biblioteche. Il libro dei fatti, la demitizzazione del passato è importantissima, ma non sempre ben accettata; non è esclusa neanche la città di Trieste, che invece da molti punti di vista sembra ultimamente pronta a vedere il proprio passato non così glorioso come invece si era abituati a considerarlo.

In questa fila di libri cruciali per il racconto del passato bisogna menzionarne uno pubblicato per la prima volta nel 1988 (la seconda edizione è del 2015) curato dallo storico triestino Elio Apih sotto il semplice titolo Trieste. Fa parte della collana fondata da Laterza editore ${ }^{6}$ di Bari intitolata Storia delle citta italiane; dopo la presentazione delle città di Firenze, Bologna, Venezia, Catania, Torino, Napoli e Reggio Calabria, si è voluta presentare la città di Trieste (la stessa collana comprende anche le città di Palermo, Padova, Perugia, Modena e Cagliari). Si doveva sempre seguire una struttura prestabilita, la quale prevedeva una parte di storia politica, sociale, economica e culturale. Oltre a Elio Apih, l'autore della storia politica e sociale, ha partecipato Giulio Sapelli, il quale ha scritto la parte inerente la storia dell'economia, mentre il terzo e per noi molto importante autore Elvio Guagnini, professore emerito di italianistica dell'Università di Trieste, ha fornito il contributo per questo libro che porta il titolo: La cultura, una fisionomia difficile. Non c'è un valido studio sulla cultura triestina che non sia marcato con segni negativi! Solo liberandosi dai miti e dalle leggende che ostacolavano la sua crescita, passando per la demitizzazione radicale, la cultura triestina ha raggiunto livelli invidiabili e oggi si può misurare con delle città che alle spalle hanno storie molto più ricche e più lunghe.

Guagnini si è concentrato abbastanza sulle differenze tra la cultura del periodo asburgico di Trieste, quando la città esprimeva il desiderio di unirsi all'Italia, e del periodo successivo al 1918, quando si sentì emarginata, appartenente ad una periferia territoriale e culturologica. Una, tra le conseguenze, è la nascita della necessità di imitare classici italiani e addirittura i modelli classicheggianti di varie forme della poesia scritta in latino. Correva l'anno 1932 quando Ferdinando Pasini, professore universitario di italianistica ravvisava per la prima volta qualche tratto della letteratura regionale e la nominava letteratura giuliana, non ancora triestina. Era Pasini, appunto, che

\footnotetext{
${ }^{6}$ Lo stesso editore, insieme con il giornale locale Il Piccolo e il comune di Trieste aveva già organizzato per tre volte $\mathrm{i}$ cicli di conferenze intitolati Lezioni di storia; ogni ciclo era composto da otto o nove lezioni, incentrate sul passato di questa città. Le lezioni si tenevano di domenica alle ore 11 presso la più ampia sala teatrale di Trieste; bisognava sempre mettersi in fila qualche ora prima per poter entrare; la sala, nonostante la capacità di duemila posti, non sempre ha permesso di accogliere tutti gli interessati. È stata perciò organizzata anche una diretta streaming di tutte le lezioni.
} 
per la prima volta menzionò Italo Svevo, sebbene gli avesse attribuito un posto ancora discreto: "quest'uomo è appena un saggio delle virtù latenti nella letteratura delle terre di confine" (Guagnini 2015: 306).

Appena Bruno Maier (Capodistria, 1922 - Trieste, 2001) in un testo pubblicato nel 1960 parla di "cultura specificamente 'triestina', con caratteristiche proprie nell'ambito della letteratura nazionale"7. Poi dovevano passare altri venti anni prima che Giorgio Voghera (Trieste, 1908 - ivi, 1999) pubblicasse una serie di saggi sotto il titolo Gli anni della psicanalisi (Pordenone, 1980) dove ha analizzato con fierezza e intelligenza alcune facce tra le più qualificanti del Novecento letterario triestino: "da un lato, quella collegata alla presenza dell'elemento ebraico [...], da un altro lato, quella che è da rapportare all'ingresso a Trieste (in un certo ambiente borghese, in alcuni scrittori) della psicanalisi" (Guagnini 2015: 312).

Oltre ad essere principalmente la città dell'amministrazione pubblica, Belgrado - come Trieste -, era anche la città del commercio e solamente dopo veniva considerata come città della cultura. Da città capitale, prima della Serbia, poi della Jugoslavia e negli ultimi vent'anni di nuovo della Serbia, Belgrado attirava la gente da tutte le parti del Paese, soprattutto dopo le grandi guerre (in seguito alle due guerre mondiali, Belgrado ha cambiato la struttura degli abitanti anche durante e dopo gli anni Novanta). Sempre, come nel caso di Trieste nell'epoca dell'esodo massiccio dopo la seconda guerra mondiale, i neoarrivati parlavano la stessa lingua, ma appartenevano a tradizioni e a culture diverse perché arrivavano dalla Croazia, dalla Bosnia, dal Kosovo... Queste rappresentano solo alcune somiglianze tra le due città confermando l'utilità del confronto tra i saggi e gli studi sulla loro cultura.

Potrebbe sembrare strano, ma controllando il catalogo della biblioteca nazionale serba risulta che sono pochi i libri di storia che sono in grado di testimoniare il passato della città di Belgrado. Tra questi, i libri di storia e storia culturale della città di Belgrado partono sempre da tempi molto lontani, documentati da scarni reperti archeologici e da testimonianze legate a miti e a leggende. Il più ambizioso progetto fu elaborato da un folto gruppo di esperti e pubblicato in tre cospicui volumi nel 1974 (Istorija Beograda I-III. Prosveta, Beograd), mentre la prima pubblicazione sulla storia di Belgrado risale all'anno 1933: Marija Ilić Agapova, Ilustrovana istorija Beograda. Il libro di Agapova fu ordinato e pubblicato dal comune di Belgrado in occasione delle celebrazioni del centoventicinquesimo anniversario della liberazione della città dagli Ottomani. I toni celebrativi non mancano, si parte comunque dall' antichità, per arrivare ai giorni nostri. La mostra Sedam hiljada godina Beograda, organizzata presso il Museo della città di Belgrado

\footnotetext{
${ }^{7}$ Maier (1960) qui citato da Guagnini (2015: 311).
} 
nel 1966, fu accompagnata da un catalogo redatto da Vladimir Kondić al quale successivamente (nel 1975) fu aggiunta un'antologia di testi (questa volta redatta da Jovan Todorović). Era molto più presente l'idea sul valore dell'antichità di per sé: sembrava che bastasse convincere il lettore che la città ha una lunga storia e già sarebbe chiaro quanto la città fosse importante nella mappa storico-geografica del mondo. Questo concetto rimane poi presente anche nella Kratka istorija Beograda di Milorad Pavić, di cui parleremo più avanti. Concetti simili, con le affermazioni romantiche sul passato culturale belgradese, si troveranno inoltre in un testo premiato dall'Accademia delle scienze di Belgrado nel 1938 e ristampato più volte nel corso degli anni Novanta e intitolato Pogled s Kalemegdana e scritto dall'allora ministro dell'educazione, Vladimir Velmar-Janković, ma anche nei saggi più recenti, come per esempio nel libro di Jovan Pejčić Kultura i pamćenje (1998).

L'unico progetto diversamente concepito, critico verso l'uso dei miti e delle leggende come prevalente fondo storico, è rimasto finora incompiuto. Nikola e Radovan Samardžić, insieme con Mirjana Roter Blagojević, hanno già due volte pubblicato il primo volume (rispettivamente nel 2014 e nel 2016) dei tre previsti, intitolati Kulturna istorija Beograda. Finanziato dal Ministero dell'educazione e della scienza, come "opera capitale" questo progetto non parte più dal lontano passato ma dal Settecento e sembra anche intenzionato a voler sfatare i miti sull'antichità intesa come valore di per sé.

Anche questo nostro saggio sarebbe diversamente strutturato se fosse già stato pubblicato questo progetto che finalmente ci sembra scritto non con lo scopo di abbellire la storia della città, ma di presentarla così com'era. In assenza di una storia culturale moderna, mettiamo al centro del nostro interesse la prosa. Seppure la letteratura ha scelto la città di Belgrado come spazio dove svolgere le trame solo a partire dal Novecento, si potrebbe comunque sostenere che la prosa belgradese si dimostra abbastanza ricca ed è proprio lì che spuntano cenni critici verso la storia stessa.

Uno dei temi centrali della prosa situata a Belgrado è rappresentato dalla guerra, dalle sofferenze, dalle devastazioni, dalle fughe dalla città e poi dal rientro e dalla ricostruzione, dai nuovi arrivi delle masse di persone nella città. Le sventure degli individui appena arrivati dalla provincia rappresentano il tema principale all'interno del primo romanzo situato a Belgrado (Milutin Uskoković, Došljaci - I neoarrivati, prima edizione nel 1910), come anche in tanti altri racconti di Simo Matavulj (Beogradske priče, prima edizione nel 1902), nel romanzo Gospođica (La signorina, prima edizione nel 1945) di Ivo Andrić... Il problema degli spostamenti massicci della gente (non sconosciuto neanche ai triestini) sarà presente anche nei romanzi di Slobodan Selenić (Prijatelji - Gli amici, prima edizione nel 1980; Očevi i oci- Padri e babbi, prima edizione nel 1985), Svetlana Velmar Janković (Lagum, 
prima edizione nel 1990), per citarne solo alcuni. Oltre ad essere distrutta e ricostruita più volte ${ }^{8}$, la città ha ripetutamente cambiato l'ordinamento sociale e ideologico, il che ha portato al cambiamento delle denominazioni delle vie e delle piazze, alle erezioni e poi ai conseguenti abbattimenti dei monumenti storici. Tutti questi cambiamenti e le revisioni della storia hanno come conseguenza la crisi dell'identità personale ${ }^{9}$. Perciò, invece di occuparsi dei temi legati ai problemi dei neoarrivati che rallentano notevolmente il processo dell'urbanizzazione così che sempre di più si scontra l'urbano con il rurale, il centro con la periferia, dagli anni Novanta diventa più frequente, come tema letterario, la crisi d'identità. Come esempio si può citare Novobeogradske priče (Racconti neobelgradesi, prima edizione nel 1994) di Mihajlo Pantić, U potpalublju di Vladimir Arsenijević, oppure il romanzo di Vladimir Pištalo Milenijum u Beogradu (Millennio a Belgrado, prima edizione nel 2000).

Sarebbe da aggiungere che nei Balcani, tradizionalmente, non c'erano grandi centri urbani, le città importanti nascono solo nel corso del Novecento. A differenza del Mediterraneo, nei Balcani le case raramente si costruivano di pietra (Bogdanović 1982: 195). Come esempio della cultura architettonica rurale, Bogdanović cita la regione centrale della Serbia, la Šumadija, dove il fiume Morava esondava spesso e portava via le casupole costruite di fango e di paglia (Bogdanović 1982: 284). Pensando a tutto ciò, capiremo molto meglio il valore simbolico che la palude ha nel primo volume del romanzo Seobe di Miloš Crnjanski. Soprattutto leggendo il romanzo insieme con una prosa odeporica dello stesso autore e pubblicata lo stesso anno, Ljubav u Toskani (Amore in Toscana), si nota che Crnjanski è affascinato dalle pietre bianche presenti negli edifici e monumenti toscani, che sono in grado di conservare e di trasmettere oggi anche a noi le tracce di culture lontanissime nel tempo, per esempio quelle etrusche. Da grande viaggiatore e uomo di alta cultura, Crnjanski si è accorto presto che la cultura europea si identificava con la cultura urbana (European landscape is a European cityscape, come spesso viene ribadito), mentre le culture balcaniche sarebbero rurali e non riuscirebbero a conservare a lungo i documenti sulla vera storia del loro territorio. Queste prose giovanili dello scrittore che diventò uno dei più grandi prosatori serbi si possono interpretare come un confronto tra la cultura europea, da una parte, e quella slava, dall'altra. La differenza non è solo architettonica (città dall'una, e centri abitati che spariscono ad ogni alluvione dall'altra), ma profondamente culturale: la pietra mantiene le tracce

${ }^{8}$ Solo durante il Novecento la città fu bombardata tre volte.

${ }^{9}$ Vedere a proposito: Bogdanović (1982), e soprattutto la nozione descritta al numero 317, come anche Rihtman Auguštin (2000: 29). 
del passato e dunque l'Europa, le città europee sono piene di memorie. Nei Balcani, invece, tutto inizia sempre da capo.

Oggi, al contrario, non si può negare che nei Balcani esistano città moderne: Belgrado, Zagabria, Sarajevo, Lubiana, Bucarest, forse anche Sofia, sono diventati centri urbani di notevole rango. Per raggiungere ciò tutte queste città, ma soprattutto Belgrado "si è avidamente precipitata nel processo della modernizzazione” (Marković 1992: 22). Studiando il caso di Belgrado, Marković sottolinea che in questa città la modernizzazione è stata parziale; solo un sottile strato dei suoi abitanti si è urbanizzato: "sono state cambiate le forme, mentre sono molto meno moderne le strutture sociali e produttive" (Marković 1992: 23).

Questa modernizzazione, descritta come scissa, solo parziale e deformata, è molto presente nella prosa su Belgrado. Già verso la metà dell'Ottocento, a Belgrado tornano studenti che hanno studiato all'estero e quando tornano, si vestono, si nutrono e abitano "da parigini" in un contesto che è ancora quasi rurale. Questi stili di vita così sproporzionati diventano materiale molto adatto alla commedia e infatti Jovan Sterija Popović, illustre commediografo serbo, scrive un ottimo pezzo teatrale, intitolato Beograd nekad i sad (Belgrado di una volta e di oggi, apparso per la prima volta nel 1853). Dopo la prima guerra mondiale tornano da Parigi quasi quattromila giovani che si erano lì scolarizzati durante e subito dopo la guerra; al loro ritorno nel Paese i cambiamenti sociali diventano ancora più veloci, superficiali, visibili nella vita politica e culturale, ma l'economia e la vita istituzionale rimangono pietrificate e gravemente manipolate. E così, ottanta anni dopo Sterija, un altro commediografo eccellente, Branislav Nušić, scrive di nuovo la commedia utilizzando lo stesso titolo (Beograd nekad i sad, 1933) e prendendo spunto dagli stessi processi di modernizzazione deformata.

Boško Tokin, uno tra i giovani che si era scolarizzato a Parigi, pubblicò il romanzo Terazije ${ }^{10}$ (1932). Tokin fu un personaggio poliedrico, scrisse poesie, si occupò molto di critica letteraria e scrisse molto riguardo ai film. Era il primo teorico e critico cinematografico in Serbia. Affascinato dalla tecnica cinematografica, vi ricorre anche nella stesura del romanzo: si susseguono i quadri ingranditi, i personaggi e i paesaggi zoomati, i vari flashback... Ma quello che conta è soprattutto l'enorme contrasto tra il centro e la periferia, tra 1'“europeismo" (falsato, superficiale) e il primitivismo. L'Autore cerca di presentare queste anomalie come conseguenze della guerra; il romanzo finisce con una scena che si svolge nel 1929, quando fu bandito il parlamento e introdotta la dittatura e dunque il romanzo copre il lasso di tempo corrispondente al primo decennio successivo alla prima guerra mondiale. Qui bisogna aggiungere che in questa zona le guerre erano numerose, così

\footnotetext{
${ }^{10}$ Terazije è il nome di un quartiere centralissimo della città di Belgrado.
} 
che fino ad oggi si può parlare di Belgrado postbellica, come anche della presenza dello scontro tra il centro e la periferia come uno dei temi centrali della letteratura belgradese ${ }^{11}$.

Ci sono poi alcune varianti di questo tema centrale: nel romanzo Lagum (1999) Svetlana Velmar Janković nota la differenza tra la classe borghese proveniente dalla Voivodina (nel romanzo rappresentata da prof. Pavlović nato a Novi Sad) e la élite belgradese che in tanti è villan rifatta. Mentre i racconti e romanzi precedenti della stessa autrice si soffermano piuttosto sui temi legati all'Ottocento, abbellendo le figure storiche del periodo, nel romanzo Lagum il patrimonio culturale e sociale affermatosi nei processi della modernizzazione e delle varie avanguardie vengono soppressi, dopo la seconda guerra mondiale, da un realismo socialista villano e sgarbato.

Anche il periodo successivo alla seconda guerra mondiale è caratterizzato dallo scontro tra il centro e la periferia, tra l'urbano e il rurale. Nel romanzo Prijatelji (Amici, 1980) di Slobodan Selenić il centro non è solo localizzato in un quartiere, ma in una via e corrisponde ad un preciso numero della via Kosančićev venac, il numero 7 (il romanzo è stato drammatizzato e messo in scena proprio con questo titolo: Kosančićev venac 7). A questo indirizzo, nel centro della città si svolgono i processi che dopo la seconda guerra mondiale hanno caratterizzano tutta la città. La casa, che apparteneva alla famiglia, era architettonicamente moderna e l'attuale proprietario Vladan Hadžislavković ha finito le scuole a Cambridge. Secondo le nuove regole le autorità nazionalizzano le case e nelle migliori (come quella di Vladan) s'insediano gli ex partigiani provenienti dalle montagne e per niente abituati alla cultura urbana. Tra Vladan e questi neoarrivati ci sono differenze culturali molto più profonde che tra lui e gli inglesi. Vladan si è laureato studiando la guerra civile inglese e perciò è in grado di notare che la storiografia inglese ha studiato e descritto molto dettagliatamente ogni piccolo particolare di quel lontano Settecento, mentre la storia serba "pare un enorme buco dove domina il buio" (Selenić 2000: 43). Nonostante abbia letto tutti i documenti disponibili, la storia del proprio popolo e della propria famiglia Vladan ha imparato ascoltando lo zio, dunque la storia gli è stata trasmessa tramite racconto orale. Perciò, quando nell'unica stanza che gli è rimasta di tutta la casa di famiglia sceglie come coinquilino il giovane Istref Veri, albanese proveniente dal Kosovo, il quale è scappato dal proprio Paese perché temeva una vendetta di sangue, in un primo momento il lettore potrebbe pensare che la scelta di Vladan sia tinta di qualche sentimento

11 "Dal 1876 fino al 1991, e dunque in centoquattordici anni la Serbia ha partecipato ad otto guerre. In media una ogni quattordici anni. Il periodo più lungo senza guerre è quello corrispondente alla seconda Jugoslavia: dal 1945 al 1991", scrisse Latinka Perović in una relazione preparata per una conferenza episcopale e pubblicata su e-Novine, 29/09/2008. 
omoerotico (che Vladan dimostra, ma non concretizza mai fisicamente). In seguito però il lettore si accorge che questa scelta avrebbe un valore allegorico: sia Vladan sia Istref conoscono solo la storia orale delle proprie famiglie e dei propri popoli. Lontani dai fatti, loro due raccontano il passato basandosi su leggende, su racconti orali, su testimonianze orali. Istref poi riesce a inserirsi nella nuova società socialista, mentre Vladan no, non solo perché è più avanti con gli anni ed è cresciuto in un altro sistema sociale, ma proprio perché Vladan sente che la tragedia che subisce il suo popolo proviene dalla poca conoscenza della storia vera, della storia dell'accaduto. L'inesperienza, la negligenza ha come conseguenza questi cambiamenti sociali rocamboleschi, dove crolla e sparisce quel sottile strato urbano e quelli che fino a ieri non sapevano neanche leggere prendono tutto nelle loro mani.

Abbiamo visto che Selenić, in questo romanzo, ha reso estremamente compatto il problema dello scontro tra il centro e la periferia, tra l'urbano e il rurale, portando lo scontro dentro la stessa casa e la stessa stanza dei due personaggi che rappresentano le nazioni che erano e sono rimaste in conflitto (serbi e kosovari). Inoltre, Selenić ha riflettuto e spiegato la mancanza di una storia affidabile, scritta sui fatti che fosse in grado di presentare oggettivamente il breve ma importante passato della vita attiva della borghesia belgradese.

Nel romanzo Milenijum u Beogradu (Millennio a Belgrado, 2000) di Vladimir Pištalo, rimarrà come centrale lo scontro generazionale ed ideologico, ma non saranno più confrontati il centro e la periferia. La tecnica della scrittura di Pištalo è sulla traccia di quella di Tokin, ma è molto più spezzettata e frammentata di quella che una volta sarebbe stata interpretata come d'avanguardia. La prosa di Pištalo cerca di presentarsi attraverso uno stile caratterizzato da leggerezza e spontaneità; come se volesse suggerirci che le cose che lui descrive corrispondono ad una prassi quotidiana.

Sarebbe difficile riassumere i quarantasette frammenti di quest'arguto, ingegnoso romanzo. Con tanta ironia prende in mira già nel prologo le leggende sull'antichità della città di Belgrado, sulla sua struttura multietnica, su tanti personaggi che avrebbero abbellito e costruito la città. Poche sono invece le tracce visibili del passato. Il prologo se la prende con il concetto che attribuisce alla città una storia lunghissima. Questo lungo passato, Pištalo lo presenta come se fosse un sogno; bisogna però subito aggiungere che nel romanzo si attribuisce un valore alto ai sogni e questi vengono trattati come se fossero realtà. Nell'epilogo, intitolato Lament nad Beogradom, omonimo al celebre poema di Miloš Crnjanski (di cui parleremo più avanti), il narratore si addormenta e nel sogno si chiede dove siano sparite tutte le fortezze, le moschee e i palazzi di cui parlano tanto le leggende. Come se questa città esistesse solo nel sogno, nelle storie orali e nelle leggende. In 
realtà, Belgrado è una città lacerata, una piaga e nel momento in cui sulla sua superficie si forma la crosta, questa viene tolta via da unghie luride, così che di nuovo si forma la piaga. Perciò le generazioni dei figli non possono mai proseguire il mestiere dei padri. È una città dove la gente irride proprio quello che desidera ardentemente, volgendo lo sguardo ai propri sogni, il che vuol dire che loro stessi con i propri caratteracci, con il proprio comportamento e con le proprie azioni dalla città costruiscono un'anti-città .

Il narratore (che si presenta sempre in prima persona) ci mostra che negli anni Novanta fosse uno tra i rari, se non addirittura l'unico in grado di vedere e di toccare da vicino le dimensioni illusorie, assolutamente inventate, della propria città. La generazione alla quale appartiene (lo scrittore è nato a Sarajevo nel 1960) ha perso tutti i punti d'appoggio, tutti i riferimenti stabili e perciò gli individui sono diventati molto solitari, incerti, sciagurati.

Il narratore è di professione uno storico (Pištalo stesso è un professore di storia che insegna negli Stati Uniti d'America) che incolpa il padre in quanto rappresentante della generazione che non ha saputo scrivere e presentare una storia critica e invece di presentare la realtà, ha descritto Belgrado "come centro del mondo" (Pištalo 2000: 120). I padri avevano scritto "una storia fantomatica. Falsificare, aggiungere le bugie riempivano loro con il senso del potere" (Pištalo 2000: 121).

Completamente diversa sarebbe l'immagine della città di Belgrado se uno prendesse tra le mani il libro Kratka istorija Beograda (Breve storia di Belgrado), pubblicato per la prima volta nel 1999 con testo a fronte tradotto in inglese. Finora sono uscite dieci edizioni di questo libro scritto dal noto e affermato scrittore e professore universitario Milorad Pavić (1929-2009). Riportiamo qui alcune frasi introduttive su "una delle più antiche e più volte distrutte città del mondo":

"Belgrado più antica, l'abitato che ancor oggi porta lo stesso nome, sebbene non si conosca il vero nome di allora, quando la città fu costruita secondo i principi della cultura neolitica, la così detta cultura di Vinča ${ }^{12}$ che si sviluppò tra il 2300 e il 2000 a. C., che produsse ed esportò le statuette di donne, di uomini e molti animali fantasiosi. Se venissero confermati alcuni calcoli recenti, Belgrado come abitato sarebbe coetaneo ad alcuni motivi della poesia popolare orale che, come oggi si presuppone, nasce durante il periodo neolitico" (traduzione mia) ${ }^{13}$.

${ }^{12}$ Nel villaggio di Vinča, situato sulle rive del Danubio, quattordici chilometri più a valle di Belgrado, venne rinvenuto un grande insediamento neolitico.

13 “Najstariji Beograd koji znamo, naselje koje još nosi ovo ime i koje je za nas ostalo bezimeno, sazdan je na osnovama najveće neolitske kulture, čuvene vinčanske kulture koja se na tom tlu javlja između 2300. i 2000. godine pre naše ere sa svojom grnčarijom uvoznom i izvoznom, sa svojim bezbrojnim statuetama ljudskih, naročito ženskih figura i fantastičnih životinja. Ako su neki najnoviji proračuni tačni, Beograd bi tako kao naselje 
Gli slavi si sono avvicinati a Belgrado nel sesto secolo ma l'autore in questione anticipa alcune tracce della loro cultura di alcuni millenni! La sintassi apodittica è senza alcuna ambiguità, come se l'autore non conoscesse la retorica letteraria. Tutto è stato formulato con l'unico scopo di rendere comprensibile la lunga vita, l'antichità della cultura serba, come se questa esistesse dal neolitico in poi. È impossibile non stupirsi di come questo autore, così colto, possa scrivere un testo linguisticamente così povero, piatto e per niente documentato, nel quale una dopo l'altra si susseguono delle semiverità. Per esempio: la conferma dell'uso della lingua serba presso la diplomazia ottomana nella capitale dell'Impero Ottomano proverebbe che "la lingua serba è ancora oggi in uso a Istanbul" (Pavić 2000: 27). Si possono leggere asserzioni come la seguente: solo quindici anni dopo la Francia, la Serbia ha vissuto la propria "rivoluzione borghese" (e si riferisce alla prima rivolta contro gli ottomani del 1804!). Come conseguenza, secondo Pavić, a "Belgrado spuntano subito i musei, le librerie, le associazioni scientifiche, artistiche e musicali, mentre la vita teatrale diventa ogni giorno più vivace" (Pavić 2000: 45). Il tempo corre accelerato, la vita culturale belgradese si evolve con una velocità che difficilmente si può immaginare. La conferma? Due teatri - «Teatar na đumruku» (dal 1841) e «Kod jelena» (dal 1847), come anche il gruppo itinerante di Pavel Đurković, attivo nella cittadina di Pančevo ${ }^{14}$ presso «Trubač» (dal 1844), che viene in tournée anche a Belgrado, fa il nucleo di questa vita teatrale. Pavić non si sbilancia davanti ai fatti: tutti questi "teatri" erano amatoriali e non hanno avuto una lunga vita. Inutilmente cercheremo i fatti: quando fu costruito il primo teatro stabile a Belgrado, quando fu fondato il festival internazionale di Belgrado (BITEF) e perché fu così importante... Se poi si passa ai dati storici, questi vengono dimostrati in un modo ancora peggiore: sembra che a Belgrado non sia passata né la prima né la seconda guerra mondiale, mentre le guerre degli anni Novanta vengono così descritte: "Nel corso della guerra civile in Croazia e in Bosnia nel mondo spuntavano le richieste di bombardare Belgrado. In quel momento storico nel 1993 a Belgrado e in Serbia erano in atto una super inflazione e tanta fame. Dai parchi pubblici sparivano le panchine di legno perché la gente li usava per accendere le stufe" (Pavić 2000: 57).

Sarebbe inutile citare altri esempi dal testo scritto da Pavić; sembra proprio che fosse lui il rappresentante della generazione dei padri che falsavano la storia, della quale parlava il narratore nel romanzo Millennio a Belgrado. Le tesi che espone non sono argomentate. L'Autore non prende in consi-

bio vršnjak nastanku najstarijih motiva srpskog usmenog stvaralaštva, koje, kako se danas misli, nastaje negde u neolitu" (Pavić 2000: 5).

${ }^{14}$ All'epoca questa piccola città faceva parte dell'Impero asburgico e dunque difficilmente poteva far parte della città di Belgrado liberatasi dagli ottomani. 
derazione il fatto che creare una geografia letteraria richieda all'autore la stessa oggettività necessaria alla geografia vera e propria; lo spazio presente nell'opera scritta non si deve sottovalutare. Una parte delle differenze tra la sintassi delle due opere sulle città, quella di Magris, autore di Microcosmi e quella di Pavić della Kratka istorija Beograda, fa parte delle differenze tra $\mathrm{i}$ due generi letterari che loro avevano scelto: Magris scrive una prosa postmoderna che difficilmente si potrebbe definire dal punto di vista del genere letterario, ma comunque piena di riflessioni, passaggi saggistici, dati e fatti concreti. Pavić invece scrive un baedeker quasi letterario e addolcito della città di Belgrado. Ma la differenza principale tra questi due scrittori è frutto delle differenze tra l'urbanità della cultura di queste due città che si assomigliano, ma sono anche tanto differenti; la differenza principale però esiste tra i punti di vista degli autori e della loro filosofia quotidiana: Magris sostiene una visione moderna, transnazionale e multiculturale del mondo, Pavić invece solo quella nazionale di vecchio stampo.

Se prendiamo il libro di Pavić come esempio negativo della saggistica che pone al centro del suo interesse la città di Belgrado, il poema Lament nad Beogradom (Lamento su Belgrado, pubblicato nel 1956) di Miloš Crnjanski rappresenta un testo letterario più raffinato e più elaborato sullo stesso tema. Si svolge seguendo due filoni paralleli e allo stesso tempo in forte contrasto: i versi di un corso, stampati in alto della pagina sinistra, e dell'altro invece in fondo della pagina destra, formano insieme le immagini più poetiche della città di Belgrado. Crnjanski scrive questo poema durante il suo esilio a Londra, quando ancora non era benvenuto a casa. I versi che si trovano sulla pagina sinistra cercano di esprimere le emozioni più intime e sono impregnati di uno stato di disperazione e di nichilismo che il poeta vive nel momento in cui scrive questa poesia. In fondo, nella pagina destra, Crnjanski riporta invece le immagini che il soggetto poetico inventa e concepisce sulla città amata, ma per lui irraggiungibile. Nelle immagini, nelle fantasie, la città cresce, si allarga sopra il fiume, si muove, respira, brilla... Abbiamo già visto che a Crnjanski piace mettere a confronto le regioni geograficamente distanti e diverse tra di loro. Anche qui lui si appoggia a questo parallelo: i sentimenti nichilistici, il presentimento della morte, le angosce, le diffidenze, tutto ciò si attribuisce all'isola lontana e deserta nel mare Antartico di nome Jan Majen. Da questa parte giungono anche i versi che descrivono i ricordi belli del passato; proprio perché il passato si presenta come morto. Era morto per il soggetto della poesia. Dalla parte sinistra, invece, scorre un inno a Belgrado. Il poeta vive già da trent'anni in esilio e immagina Belgrado come il rifugio che verrà dopo la morte. Allora Belgrado diventerà la serenità, la luce, la crescita e la brillantezza, ma solo allora, dopo la morte del soggetto poetico. La bellezza e la fastosità della 
città appartengono ad un futuro immaginato; il presente e il passato invece avevano condotto il soggetto poetico fino ad un tale punto di delusione che nella sua mente gli era attraversato addirittura il pensiero del suicidio. In questa posizione esistenziale, quando né il presente né il passato non possano avere nessun valore, il bello è presente solo nel paradiso, dopo la morte, e questo paradiso per il poeta corrisponde proprio alla Belgrado immaginata.

Vorrei che questa prova di studi paralleli tra le immagini letterarie delle due città stimolassero delle domande e incoraggiassero la nascita di saggi simili. Tra le possibili conclusioni ne sceglierei solo una: la città di carta si può costruire solo con una scrittura sensibile, complessa, affidabile. Con la parola, con il sentimento urbano. Finché si cerca di abbellire, di semplificare, di aver poca cura per i fatti, nascono le leggende, i miti, la storia orale. Non quella critica.

\section{BIBLIOGRAFIA}

AA.VV. (1974). Istorija Beograda I-III. Beograd: Prosveta.

Andrić, I. (1945). Gospođica. Beograd: Prosveta.

Apih, E. (a cura di). (2015). Trieste. Roma-Bari: Editori Laterza.

Ara, A. \& Magris, C. (1982). Trieste, un identità di frontiera. Torino: Einaudi.

Arsenijević, V. (1995). U potpalublju. Beograd: Vreme knjige.

Betizza, E. (1958). Il fantasma di Trieste. Milano: Longanesi.

Bogdanović, B. (1982). Gradoslovar. Beograd: Vuk Karadžić / Kulturno prosvetna zajednice Srbije.

Bonazza, S. (1990). Dositej Obradović i italijanska kultura. Naučni sastanak slavista u Vukove dane, 19, 2, 312-328.

Crnjanski, M. (1929a). Ljubav u Toskani. Beograd: Izdavačka knjižarnica Gece Kona.

Crnjanski, M. (1929b). Seobe. Beograd: Izdavačka knjižarnica Gece Kona.

Crnjanski, M. (1983). Lament nad Beogradom. In M. Crnjanski, Pesme. Dela Miloša Crnjanskog. Vol. I (pp. 263-269). Beograd: Nolit.

Fölkel, F. \& Cergoly, C. (1983). Trieste provincia imperiale. Milano: Bompiani.

Guagnini, E. (2015). La cultura, una fisionomia difficile. In E. Apih (a cura di), Trieste (pp. 271-393). Roma-Bari: Editori Laterza.

Ilić Agapova, M. (1933). Ilustrovana istorija Beograda. Beograd: Biblioteka opštine grada Beograda.

Karadžić, Vuk Stefanović (1935). Srpski rječnik, istumačen njemačkijem $i$ latinskijem riječima. Beograd: Stamparija Kraljevine Jugoslavije. 
Magris, C. (1997). Microcosmi. Milano: Garzanti.

Maier, B. (1960). Condizione della letteratura triestina del Novecento. L'Approdo letterario, 9, n. s., a. VI, gennaio-marzo, 5-9.

Marković, J. P. (1992). Beograd i Evropa 1918-1941. Beograd: Savremena administracija.

Matavulj, S. (1996). Beogradske priče. Beograd: BMG.

Millo, A. (2007). Ferruccio Fölkel: un intellettuale triestino tra storia e letteratura. Nuova Corvina, 19, 78-92. Testo disponibile al sito: http://epa.oszk.hu/02500/02582/00019/pdf/EPA02582_nuova_corvina_2007_19_078-092.pdf (10/10/2016)

Mitrović, M. (2008). Trst Dositejevog doba. In D. Ivanić \& V. Jelić (a cura di), Delo Dositeja Obradovića 1807-2007 (pp. 361-370). Beograd: Zadužbina "Dositej Obradović".

Mitrović, M. (2009a). Dositej e Trieste: la poetica e il contesto teorico. Ricerche slavistiche. Nuova serie. Vol. 7, LIII, 72-93.

Mitrović, M. (2009b). La poetica del libro triestino di Dositej Obradovic. In M. Mitrović (a cura di), Cultura serba a Trieste (pp. 87-110). Lecce: Argo.

Mitrović, M. (2011). Značaj prostora u poetici Mezimca. In D. Ivanić (a cura di), Zbornik radova: Naučni skup Dositej i (srpska) škola (pp. 181-191). Beograd: Zadužbina "Dositej Obradović".

Mitrović, M. (2013). Trst u Dositejevo doba. Dositejev vrt. Godišnjak Zadužbine "Dositej Obradović”, 1, 17-35.

Nušić, B. (2006). Beograd nekad i sad. In B. Nušić, Sabrana dela. Vol. 11 (pp. 283-330). Beograd: Prosveta.

Obradović, D. (2008). Mezimac (Sabrana dela. Vol. IV). Beograd: Zadužbina "Dositej Obradović".

Pantić, M. (1994). Novobeogradske priče. Beograd: Vreme knjige.

Pavić, M. (2000). Kratka istorija Beograda. Beograd: Dereta.

Pejčić, J. (1998). Kultura i pamćenje. Beograd: Stubovi kulture.

Pellegrini, E. (1987). La Trieste di carta: aspetti della letteratura triestina del Novecento. Bergamo: P. Lubrina.

Pellegrini, E. (2002). La città di carta. In R. Finzi, C. Magris \& G. Miccoli (a cura di), Storia d'Italia. Le Regioni. Dall'unità ad oggi. Il Friuli Venezia Giulia, II (pp. 1205-1216). Torino: Einaudi.

Pištalo, V. (2000). Milenijum u Beogradu. Beograd: Narodna knjiga / Alfa.

Popović, J. S. (2005). Beograd nekad i sad. In J. S. Popović, Pomirenja (pp. 45-57). Vršac: Književna opština Vršac.

Rihtman Auguštin, D. (2000). Ulice moga grada. Beograd: Biblioteka XX vek.

Samardžić, N., Samardžić, R. \& Roter Blagojević, M. (2014). Kulturna istorija Beograda: 18. vek. Beograd: Hesperia edu. 
Selenić, S. (2000). Prijatelji. Beograd: Prosveta.

Selenić, S. (2009). Očevi i oci. Beograd: Laguna.

Tokin, B. (1932). Terazije. Beograd: Izdavačka knjižarnica Gece Kona.

Uskoković, M. (1966). Došljaci. Sarajevo: Svjetlost.

Velmar Janković, S. (1999). Lagum. Beograd: Stubovi kulture.

Velmar-Janković, V. (1938). Pogled s Kalemegdana. Beograd: Gregorić.

\section{CITY OF PAPER}

\section{Summary}

This article traces the long journey that brought to Trieste its title of City of Paper, and it compares the literary image of Trieste to that of Belgrade. Even though writers certainly contributed to the construction and composition of the literary profile of Trieste, and helped turning it into a major cultural centre of the 20th century, all this would have been impossible without a strong "logistic" support from literary historians and critics (Ara, Magris, Fölkel, Guagnini). In the last decades, the city's plural identity became visible. And it was only through liberation from myths and legends that the culture of Trieste was able to reach such an enviable level, being today on an equal footing with cities whose history is much richer and much longer. On the other hand, history and cultural history books dedicated to Belgrade always begin from a very distant past, documented by scarce archeological findings and evidence related to myths and legends (Velmar-Janković, Pavić, Pejčić). Given that a modern cultural history is missing, we focus on novels that take a critical look at Belgrade's cultural history (Uskoković, Matavulj, Andrić, Crnjanski, Velmar-Janković, Selenić, Pantić, Pištalo).

Keywords: Trieste, Belgrade, modern cultural history, literature, demythisation, plural identity. 